\title{
Bessere Ergebnise bei MRT-Kontrolle?
}

Fragestellung: Ist eine MRT-basierte interventionelle Therapie des ischämischen Insults einer systemischen Thrombolyse mit rt-PA überlegen?

Hintergrund: Die im Moment einzig verfügbare und zugelassene Therapie des akuten ischämischen Insults ist die systemische Thrombolyse mit rt-PA im 4,5 Stunden-Zeitfenster. In drei deutschen Stroke-Units erhalten etwa 10-15\% aller Patienten diese Therapie. Seit mehreren Jahren stehen jetzt endovaskuläre Therapieverfahren zur Verfügung mit denen eine höhere Rekanalisierungsrate bei M1-Verschlüssen erreicht wird als mit der systemischen Thrombolyse. Ein mögliches Auswahlkriterium um Patienten mit einer Thrombektomie zu behandeln ist das Schlaganfall-MRT in dem Patienten identifiziert werden können, die eine Penumbra haben, das heißt bei denen noch potenziell zu rettendes Hirngewebe vorhanden ist.

Patienten und Methodik: In diese in den USA durchgeführte randomisierte Studie wurden Patienten innerhalb eines 8-Stunden-Zeitfensters randomisiert und erhielten entweder eine Standardtherapie einschließlich systemischer Gabe von rt-PA oder eine mechanische Thrombektomie mit dem Merci-Retriever oder dem Penumbra-System. Bei allen Patienten wurden vor der Behandlung ein CT und ein MRT durchgeführt. Die Randomisierung erfolgte stratifiziert je nachdem ob die Patienten eine Penumbra in der Bildgebung hatten oder nicht. Der primäre Outcome war eine Shift-Analyse der modifizierten Rankin-Skala nach 90 Tagen.

Ergebnisse: Die Studie begann 2004 und endete 2011. Insgesamt wurden 118 auswertbare Patienten in die Studie eingeschlossen. In der Interventionsgruppe hatten 34 Patienten eine Penumbra

Kidwell CS, Jahan R, Gornbein $J$ et al; the MR RESCUE Investigators. A trial of imaging selection and endovascular treatment for ischemic stroke. N Engl J Med 2013; 368: 914-23 und 30 keine. In der konservativen Therapiegruppe hatten 34 eine Penumbra und 20 keine. Die Patienten waren im Mittel 64 Jahre alt und hatten einen mittleren NIHSS-Score von 17. Bei 20 Patienten lag ein distaler Verschluss der

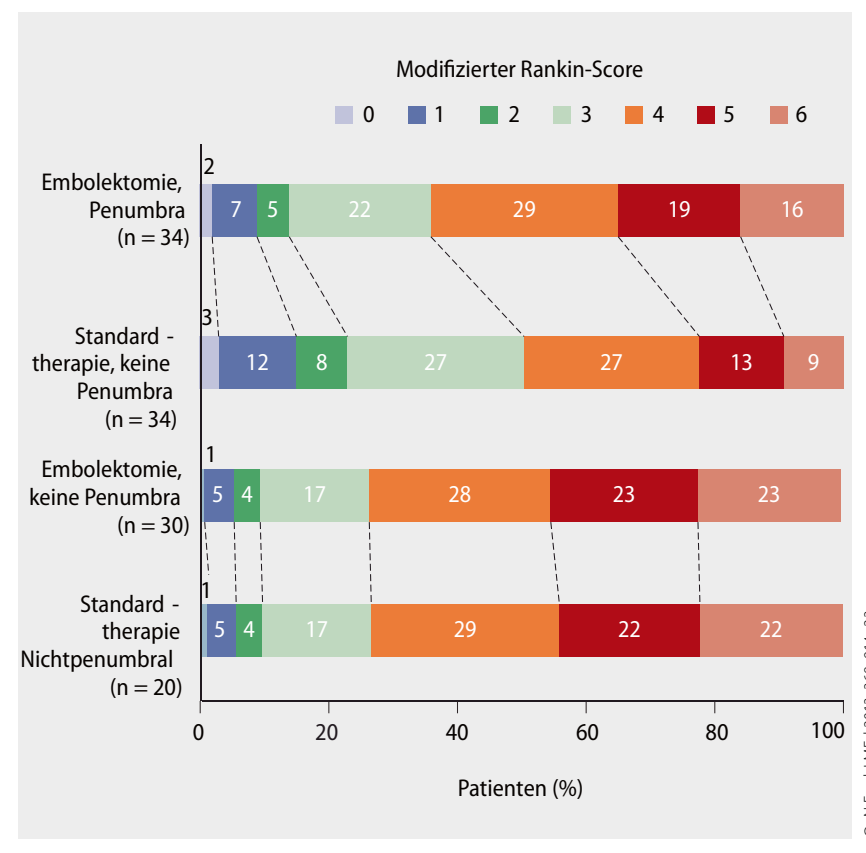

1 Funktioneller Outcome nach 90 Tagen, gemessen mithilfe der modifizierten Rankin-Skala.

Arteria carotis interna vor, bei 78 ein M1-Verschluss und bei 20 ein M2-Verschluss. Die mittlere Zeit bis zum Studieneinschluss betrug 5,5 Stunden. In der Embolektomiegruppe wurde eine Rekanalisierungsrate von $67 \%$ erreicht. Es gab keinerlei Unterschiede für primäre und sekundäre Endpunkte. Die Sterblichkeit nach 90 Tagen betrug $21 \%$ und die Rate symptomatischer intrakranieller Blutungen 4\%. Auch bezüglich der modifizierten RankinSkala ergaben sich keine Unterschiede ( Abb. 1). Es bestanden auch keine Unterschiede in Abhängigkeit davon, ob die Patienten eine Penumbra hatten oder nicht.

Schlussfolgerungen: In der MR-RESCUE-Studie ist eine lokale Thrombektomie mit dem Merci- oder Penumbra-Device einer systemischen Thrombolyse mit rt-PA nicht überlegen.

\section{- Kommentar von Hans-Christoph Diener, Essen}

\section{Ob die Bildgebung entbehrlich ist, bleibt noch offen}

Diese in den USA durchgeführte Studie hat auf den ersten Blick ein sehr attraktives Studiendesign, da sie Patienten danach stratifizierte, ob sie in der Bildgebung eine Penumbra hatten oder nicht. Die Studie hat allerdings auch sehr viele Schwächen. Zum Ersten dauerte es sieben Jahre, um 127 Patienten einzuschließen. Zum Zweiten war die Dauer bis zur Thrombektomie mit durchschnittlich 5,5 Stunden viel zu lang. Zum Dritten wurden Devices wie Merci oder Penumbra eingesetzt, von denen man in der Zwischenzeit weiß, dass die Rekanalisierungsraten im Vergleich mit den Solitaire- und TrevoDevices deutlich geringer sind. Ob aus der MR-RESCUE-Studie geschlossen werden kann, dass für zukünftige Interventionsstudien das Schlaganfall-CT oder-MRT mit Diffusions- und Perfusionsmessung entbehrlich ist, kann erst abgeschätzt werden, wenn entsprechende Studien mit modernen Devices vorliegen. 